\title{
SOLITON EQUATIONS EXHIBITING PFAFFIAN SOLUTIONS
}

\author{
RYOGO HIROTA, MASATAKA IWAO \\ School of Science and Engineering, Waseda University, Tokyo 162, Japan \\ e-mail:iwao@hirota.info.waseda.ac.jp \\ and SATOSHI TSUJIMOTO \\ Faculty of Engineering Science, Osaka University, Osaka 560, Japan \\ e-mail: tujimoto@sigmath.es.osaka-u.ac.jp
}

(Received 22 December, 1999)

\begin{abstract}
Soliton equations whose solutions are expressed by Pfaffians are briefly discussed. Included are a discrete-time Toda equation of BKP type, a modified Toda equation of BKP type, a coupled modified KdV equation and a coupled modified $\mathrm{KdV}$ equation of derivative type.
\end{abstract}

1991 Mathematics Subject Classification. 35Q51.

1. Introduction. A Pfaffian is the square root of an anti-symmetric determinant of order $2 \mathrm{n}$ :

$$
\operatorname{det}\left|a_{j k}\right|_{1 \leq j, k \leq 2 n}=\left[\operatorname{pf}\left(a_{1}, a_{2}, \cdots, a_{2 n}\right)\right]^{2} .
$$

We have, for example, for $n=1$

$$
\left|\begin{array}{cc}
0 & a_{12} \\
a_{21} & 0
\end{array}\right|=\operatorname{pf}\left(a_{1}, a_{2}\right)^{2} .
$$

Hence

$$
\operatorname{pf}\left(a_{1}, a_{2}\right)=a_{12}
$$

For $n=2$,

$$
\left|\begin{array}{cccc}
0 & a_{12} & a_{13} & a_{14} \\
a_{21} & 0 & a_{23} & a_{24} \\
a_{31} & a_{32} & 0 & a_{34} \\
a_{41} & a_{42} & a_{43} & 0
\end{array}\right|=\left[a_{12} a_{34}-a_{13} a_{24}+a_{14} a_{23}\right]^{2}
$$

Hence

$$
\operatorname{pf}\left(a_{1}, a_{2}, a_{3}, a_{4}\right)=a_{12} a_{34}-a_{13} a_{24}+a_{14} a_{23} .
$$

A square root of a determinant of a matrix $M$ of order $\mathrm{n}$ which is a sum of a unit matrix $E$ and a product of anti-symmetric matrices $A$ and $B$ is expressed by a twocomponent Pfaffian: 


$$
\operatorname{det}|E+A \times B|=\left[\operatorname{pf}\left(a_{1}, a_{2}, \cdots, a_{n}, b_{1}, b_{2}, \cdots, b_{n}\right)\right]^{2},
$$

where

$$
\begin{aligned}
& \operatorname{pf}\left(a_{j}, a_{k}\right)=a_{j k}=-a_{k j}, \\
& \operatorname{pf}\left(b_{j}, b_{k}\right)=b_{j k}=-b_{k j}, \\
& \operatorname{pf}\left(a_{j}, b_{k}\right)=\delta_{j, k}
\end{aligned}
$$

and where $\delta_{j, k}$ is Kronecker's delta symbol.

We have, for example, for $n=3$

$$
\begin{gathered}
E+A \times B=\left(\begin{array}{lll}
1 & 0 & 0 \\
0 & 1 & 0 \\
0 & 0 & 1
\end{array}\right)+\left(\begin{array}{ccc}
0 & a_{12} & a_{13} \\
a_{21} & 0 & a_{23} \\
a_{31} & a_{32} & 0
\end{array}\right)\left(\begin{array}{ccc}
0 & b_{12} & b_{13} \\
b_{21} & 0 & b_{23} \\
b_{31} & b_{32} & 0
\end{array}\right) \\
=\left(\begin{array}{ccc}
1+a_{12} b_{21}+a_{13} b_{31} & a_{13} b_{32} & a_{12} b_{23} \\
a_{23} b_{31} & 1+a_{21} b_{12}+a_{23} b_{32} & a_{21} b_{13} \\
a_{32} b_{21} & a_{31} b_{12} & 1+a_{31} b_{13}+a_{32} b_{23}
\end{array}\right) .
\end{gathered}
$$

Accordingly we find

$$
\operatorname{det}|E+A \times B|=\left[-1+a_{12} b_{12}+a_{13} b_{13}+a_{23} b_{23}\right]^{2} .
$$

On the other hand using the expansion rule of the Pfaffian

$$
\operatorname{pf}\left(c_{1}, c_{2}, \cdots, c_{2 n}\right)=\sum_{j=1}^{2 n}(-1)^{j-1} \operatorname{pf}\left(c_{1}, c_{j}\right) \operatorname{pf}\left(c_{2}, c_{3}, \cdots, \hat{c}_{j}, \cdots, c_{2 n}\right)
$$

we obtain

$$
\operatorname{pf}\left(a_{1}, a_{2}, a_{3}, b_{1}, b_{2}, b_{3}\right)=-1+a_{12} b_{12}+a_{13} b_{13}+a_{23} b_{23} .
$$

Hence

$$
\operatorname{det}|E+A \times B|=\operatorname{pf}\left(a_{1}, a_{2}, a_{3}, b_{1}, b_{2}, b_{3}\right)^{2} .
$$

The two-component Pfaffian plays an important role in expressing soliton solutions of the coupled modified $\mathrm{KdV}$ equations and coupled modified equation of derivative type described below.

We shall show that soliton solutions of the following equations are expressed by Pfaffians.

(i) Discrete-time Toda equation of BKP type

$$
\begin{aligned}
& W_{n}^{m+1}-W_{n}^{m-1}=\log \frac{\delta^{2}\left[\exp \left(W_{n+1}^{m}-V_{n}^{m}\right)+\exp \left(V_{n}^{m}-W_{n}^{m}\right)\right]+\left(1-2 \delta^{2}\right)}{\delta^{2}\left[\exp \left(W_{n}^{m}-V_{n-1}^{m}\right)+\exp \left(V_{n-1}^{m}-W_{n-1}^{m}\right)\right]+\left(1-2 \delta^{2}\right)}, \\
& V_{n}^{m+1}-V_{n}^{m}=W_{n+1}^{m}-W_{n}^{m} .
\end{aligned}
$$


(ii) Modified Toda equation of BKP type

$$
\begin{aligned}
& \frac{d}{d t} \log \frac{\beta+V_{n}}{\beta-\alpha\left(I_{n}+I_{n-1}\right)}=I_{n}-I_{n+1}, \\
& \frac{d}{d t} I_{n}=V_{n-1}-V_{n} .
\end{aligned}
$$

(iii) Coupled modified $\mathrm{KdV}$ equation

$$
\frac{\partial}{\partial t} v_{j}+6\left[\sum_{1 \leq j<k \leq N} c_{j k} v_{j} v_{k}\right] \frac{\partial v_{j}}{\partial x}+\frac{\partial^{3} v_{j}}{\partial x^{3}}=0, \quad j=1,2, \ldots, N .
$$

(iv) Coupled modified $\mathrm{KdV}$ equation of derivative type

$$
\frac{\partial}{\partial t} v_{j}+6\left[\sum_{1 \leq j<k \leq N} c_{j k} D_{x} v_{j} \cdot v_{k}\right] \frac{\partial v_{j}}{\partial x}+\frac{\partial^{3} v_{j}}{\partial x^{3}}=0, \quad j=1,2, \ldots, N .
$$

We have the discrete KP equation

$$
\left[z_{1} \exp \left(D_{1}\right)+z_{2} \exp \left(D_{2}\right)+z_{3} \exp \left(D_{3}\right)\right] f \cdot f=0
$$

where $D_{1}, D_{2}, D_{3}$ and $z_{1}, z_{2}, z_{3}$ are bilinear operators and constants, respectively.

Soliton solutions to the discrete KP equation are known to be expressed by determinants [1].

While the discrete BKP equation is expressed by

$$
\left[z_{1} \exp \left(D_{1}\right)+z_{2} \exp \left(D_{2}\right)+z_{3} \exp \left(D_{3}\right)+z_{4} \exp \left(D_{4}\right)\right] f \cdot f=0
$$

where $D_{1}, D_{2}, D_{3}, D_{4}$ and $z_{1}, z_{2}, z_{3}, z_{4}$ are bilinear operators and constants, respectively, satisfying the relations

$$
\begin{aligned}
D_{1}+D_{2}+D_{3}+D_{4} & =0, \\
z_{1}+z_{2}+z_{3}+z_{4} & =0 .
\end{aligned}
$$

Solutions to the discrete BKP equation are well parametrized by parameters $a, b$ and $c$ which are the intervals of the coordinates $l, m, n[2]$. We rewrite the discrete BKP equation using these parameters:

$$
\begin{aligned}
& (a+b)(a+c)(b-c) \tau(l+1, m, n) \tau(l, m+1, n+1) \\
& \quad+(b+c)(b+a)(c-a) \tau(l, m+1, n) \tau(l+1, m, n+1) \\
& \quad+(c+a)(c+b)(a-b) \tau(l, m, n+1) \tau(l+1, m+1, n) \\
& \quad+(a-b)(b-c)(c-a) \tau(l, m, n) \tau(l+1, m+1, n+1)=0 .
\end{aligned}
$$

Solutions are expressed by the Pfaffian $[2,3]$ :

$$
\tau(l, m, n)=\operatorname{pf}(1,2,3, \cdots, 2 N),
$$


where the elements of the Pfaffian are

$$
\begin{aligned}
& \operatorname{pf}(j, k) \equiv c_{j k} \\
& +\sum_{s=-\infty}^{n-1}\left[\phi_{j}(l, m, s+1) \phi_{k}(l, m, s)-\phi_{j}(l, m, s) \phi_{k}(l, m, s+1)\right]
\end{aligned}
$$

and $\phi_{j}(l, m, n)$ are a linear combination of exponential functions in discrete space

$$
\left(\frac{1-a p_{\mu}}{1+a p_{\mu}}\right)^{l}\left(\frac{1-b p_{\mu}}{1+b p_{\mu}}\right)^{m}\left(\frac{1-c p_{\mu}}{1+c p_{\mu}}\right)^{n}
$$

namely

$$
\phi_{j}(l, m, n)=\sum_{\mu} \alpha_{j \mu}\left(\frac{1-a p_{\mu}}{1+a p_{\mu}}\right)^{l}\left(\frac{1-b p_{\mu}}{1+b p_{\mu}}\right)^{m}\left(\frac{1-c p_{\mu}}{1+c p_{\mu}}\right)^{n} .
$$

2. Soliton equations generated by the bilinear BKP equation. The following are well-known soliton equations generated by the discrete BKP equation.

(a) Sawada-Kotera equation

$$
\begin{aligned}
& D_{x}\left(D_{t}+D_{x}^{5}\right) f \cdot f=0, \\
& u=2 \frac{\partial^{2}}{\partial x^{2}} \log f \\
& u_{t}+15\left(u^{3}+u u_{x x}\right)_{x}+u_{x x x x x}=0 .
\end{aligned}
$$

(b) Model equation of shallow-water wave

$$
\begin{aligned}
& D_{x}\left(D_{t}-D_{t} D_{x}^{2}+D_{x}\right) f \cdot f=0, \\
& u=2 \frac{\partial^{2}}{\partial x^{2}} \log f \\
& u_{t}-u_{x x t}-3 u u_{t}+3 u_{x} \int_{x}^{\infty} u_{t} d x^{\prime}=0 .
\end{aligned}
$$

Here we add two more examples.

(1) Discrete-time Toda equation of BKP type.

Let

$$
\begin{aligned}
& D_{1}=\frac{3}{2} \delta D_{t}, \quad z_{1}=1, \\
& D_{2}=-\frac{1}{2} \delta D_{t}, \quad z_{2}=-1+2 \delta^{2}, \\
& D_{3}=D_{n}-\frac{1}{2} \delta D_{t}, \quad z_{3}=-\delta^{2}, \\
& D_{4}=-D_{n}-\frac{1}{2} \delta D_{t}, \quad z_{4}=-\delta^{2} .
\end{aligned}
$$

Then

$$
\begin{aligned}
& {\left[e^{\delta D_{t}+\frac{1}{2} \delta D_{t}}+e^{-\delta D_{t}+\frac{1}{2} \delta D_{t}}-2 e^{-\frac{1}{2} \delta D_{t}}\right.} \\
& \left.\quad-\delta^{2}\left(e^{D_{n}-\frac{1}{2} \delta D_{t}}+e^{-D_{n}-\frac{1}{2} \delta D_{t}}-2 e^{-\frac{1}{2} \delta D_{t}}\right)\right] f \cdot f=0,
\end{aligned}
$$


which is rewritten as

$$
\cosh \frac{1}{2} \delta D_{t}\left[\sinh ^{2} \frac{1}{2} \delta D_{t}-\delta^{2} \sinh ^{2} \frac{1}{2} D_{n}\right] f \cdot f=0 .
$$

The bilinear form indicates that the 1-soliton solution is the same as that of the discrete-time Toda equation

$$
\left[\sinh ^{2} \frac{1}{2} \delta D_{t}-\delta^{2} \sinh ^{2} \frac{1}{2} D_{n}\right] f \cdot f=0 .
$$

The bilinear equation (1) is transformed into the nonlinear difference-difference equation in the ordinary form

$$
\begin{aligned}
& W_{n}^{m+1}-W_{n}^{m-1}=\log \frac{\delta^{2}\left[\exp \left(W_{n+1}^{m}+V_{n}^{m}\right)+\exp \left(V_{n}^{m}-W_{n}^{m}\right)\right]+\left(1-2 \delta^{2}\right)}{\delta^{2}\left[\exp \left(W_{n}^{m}+V_{n-1}^{m}\right)+\exp \left(V_{n-1}^{m}-W_{n-1}^{m}\right)\right]+\left(1-2 \delta^{2}\right)}, \\
& V_{n}^{m+1}-V_{n}^{m}=W_{n+1}^{m}-W_{n}^{m},
\end{aligned}
$$

through a series of dependent variable transformations

$$
\begin{aligned}
f_{n}^{m} & =e^{\phi_{n}^{m},} \\
\Delta_{m} \phi_{n}^{m} & =T_{n}^{m}, \\
\Delta_{n} \phi_{n}^{m} & =S_{n}^{m}, \\
\Delta_{m} S_{n}^{m} & =W_{n}^{m}, \\
\Delta_{n} S_{n}^{m} & =V_{n}^{m},
\end{aligned}
$$

where $\Delta$ is the forward difference operator defined by

$$
\begin{aligned}
& \Delta_{m} f_{n}^{m}=\delta^{-1}\left[f_{n}^{m+1}-f_{n}^{m}\right], \\
& \Delta_{n} f_{n}^{m}=f_{n+1}^{m}-f_{n}^{m} .
\end{aligned}
$$

(2) Modified Toda equation of BKP type.

Let

$$
\begin{aligned}
& D_{1}=\frac{1}{2}\left(\delta D_{x}+\epsilon D_{y}\right), \quad z_{1}=1, \\
& D_{2}=\frac{1}{2}\left(\delta D_{x}-\epsilon D_{y}\right), \quad z_{2}=-1+\beta \delta \epsilon, \\
& D_{3}=-D_{n}-\frac{1}{2}\left(\delta D_{x}+\epsilon D_{y}\right), \quad z_{3}=-\alpha \epsilon-\beta \delta \epsilon, \\
& D_{4}=D_{n}-\frac{1}{2}\left(\delta D_{x}-\epsilon D_{y}\right), \quad z_{4}=\alpha \epsilon .
\end{aligned}
$$

Then

$$
\begin{aligned}
& \left\{e^{\frac{1}{2}\left(\delta D_{x}+\epsilon D_{y}\right)}-e^{\frac{1}{2}\left(\delta D_{x}-\epsilon D_{y}\right)}\right. \\
& \quad+\alpha \epsilon\left[e^{D_{n}-\frac{1}{2}\left(\delta D_{x}-\epsilon D_{y}\right)}-e^{-D_{n}-\frac{1}{2}\left(\delta D_{x}+\epsilon D_{y}\right)}\right] \\
& \left.\quad+\beta \delta \epsilon\left[e^{\frac{1}{2}\left(\delta D_{x}-\epsilon D_{y}\right)}-e^{-D_{n}-\frac{1}{2}\left(\delta D_{x}+\epsilon D_{y}\right)}\right]\right\} f \cdot f=0,
\end{aligned}
$$

which is rewritten as 


$$
\begin{aligned}
& 2 \delta^{-1} \epsilon^{-1} \sinh \frac{1}{2} \delta D_{x} \sinh \frac{1}{2} \epsilon D_{y} f_{n} \cdot f_{n} \\
& \quad-\alpha \delta^{-1} e^{\epsilon D_{y}} \sinh \frac{1}{2} \delta D_{x} f_{n+1} \cdot f_{n-1} \\
& \quad-\beta\left[e^{\frac{1}{2}\left(\delta D_{x}+\epsilon D_{y}\right)} f_{n+1} \cdot f_{n-1}-e^{\frac{1}{2}\left(\delta D_{x}-\epsilon D_{y}\right)} f_{n} \cdot f_{n}\right]=0 .
\end{aligned}
$$

In the limit of small $\delta, \epsilon$ we have

$$
D_{x} D_{y} f_{n} \cdot f_{n}=\alpha D_{x} f_{n+1} \cdot f_{n-1}+\beta\left(f_{n+1} f_{n-1}-f_{n}^{2}\right) .
$$

The bilinear equation is transformed into the ordinary form

$$
\begin{aligned}
& \frac{\partial}{\partial x} \log \frac{\beta+V_{n}}{\beta-\alpha\left(I_{n}+I_{n+1}\right)}=I_{n}-I_{n+1}, \\
& \frac{\partial}{\partial y} I_{n}=V_{n-1}-V_{n},
\end{aligned}
$$

through the transformation

$$
\begin{aligned}
f_{n} & =e^{S_{n}}, \\
V_{n} & =\frac{\partial^{2}}{\partial x \partial y} S_{n}, \\
I_{n} & =\frac{\partial}{\partial x}\left(S_{n-1}-S_{n}\right),
\end{aligned}
$$

which we call the modified Toda equation of BKP type.

The bilinear form of the modified Toda equation of BKP type

$$
D_{x} D_{y} f_{n} \cdot f_{n}=\alpha D_{x} f_{n+1} \cdot f_{n-1}+\beta\left(f_{n+1} f_{n-1}-f_{n}^{2}\right),
$$

suggests that a new equation may be obtained by replacing the term

$$
f_{n+1} f_{n-1}-f_{n}^{2}
$$

by

$$
D_{x} f_{n+1} \cdot f_{n-1} \text {. }
$$

We shall replace the term $g_{j} g_{k}$ by $D_{x} g_{j} \cdot g_{k}$ in order to obtain a coupled modified $\mathrm{KdV}$ equation of derivative type.

The well-known modified $\mathrm{KdV}$ equation

$$
\frac{\partial}{\partial t} v+6 v^{2} \frac{\partial}{\partial x} v+\frac{\partial^{3}}{\partial x^{3}} v=0
$$

is transformed into the bilinear form

$$
\begin{aligned}
& \left(D_{t}+D_{x}^{3}\right) f \cdot g=0, \\
& D_{x}^{2} f \cdot f=2 g^{2}
\end{aligned}
$$


through the dependent variable transformation

$$
v=\frac{g}{f} .
$$

A coupled modified $\mathrm{KdV}$ equation is obtained by considering the following coupled form of the modified $\mathrm{KdV}$ equations [4].

$$
\left\{\begin{array}{l}
\left(D_{t}+D_{x}^{3}\right) f \cdot g_{j}=0, \\
D_{x}^{2} f \cdot f=2 \sum_{1 \leq j<k \leq N} c_{j k} g_{j} g_{k},
\end{array} \quad \text { for } j=1,2, \cdots N\right.
$$

which is transformed into the ordinary form

$$
\frac{\partial}{\partial t} v_{j}+6\left[\sum_{1 \leq j<k \leq N} c_{j k} v_{j} v_{k}\right] \frac{\partial v_{j}}{\partial x}+\frac{\partial^{3} v_{j}}{\partial x^{3}}=0
$$

through the transformation

$$
v_{j}=\frac{g_{j}}{f} .
$$

The multisoliton solution to equation (2) is expressed by two-component Pfaffians [4]:

$$
\begin{aligned}
f & =\operatorname{pf}\left(a_{1}, a_{2}, \cdots, a_{L}, b_{1}, b_{2}, \cdots, b_{L}\right), \\
g_{j} & =\operatorname{pf}\left(d_{0}, a_{1}, a_{2}, \cdots, a_{L}, b_{1}, b_{2}, \cdots, b_{L}, c_{j}\right), \text { for } j=1,2, \cdots, N,
\end{aligned}
$$

where the elements of Pfaffians are defined as follows

$$
\begin{aligned}
& \operatorname{pf}\left(d_{n}, a_{v}\right) \equiv \frac{\partial^{n}}{\partial x^{n}} \exp \left(\eta_{v}\right), \text { for } n=0,1,2,3, \ldots, \\
& \operatorname{pf}\left(a_{\mu}, a_{v}\right) \equiv \frac{p_{\mu}-p_{v}}{p_{\mu}+p_{v}} \exp \left(\eta_{\mu}+\eta_{v}\right), \\
& \operatorname{pf}\left(a_{\mu}, b_{v}\right) \equiv \delta_{\mu, v}, \\
& \operatorname{pf}\left(b_{\mu}, b_{v}\right) \equiv-\frac{c_{j k}}{p_{\mu}^{2}-p_{v}^{2}}, \quad\left\{\begin{array}{l}
b_{\mu} \in B_{j} \\
b_{v} \in B_{k}
\end{array}\right\}, \\
& \operatorname{pf}\left(b_{\mu}, c_{j}\right) \equiv \begin{cases}1, & \text { if } b_{\mu} \in B_{j}, \\
0, & \text { if } b_{\nu} \notin B_{k}\end{cases} \\
& \operatorname{pf}(\text { otherwise }) \equiv 0 .
\end{aligned}
$$

A coupled modified $\mathrm{KdV}$ equation of derivative type is obtained by replacing the coupling terms of the product form

$$
c_{j k} g_{j} g_{k}, \quad\left(c_{j k}=c_{k j}\right)
$$


by coupling terms of derivative type

$$
c_{j k} D_{x} g_{j} \cdot g_{k}, \quad\left(c_{j k}=-c_{k j}\right) .
$$

We obtain

$$
\begin{cases}\left(D_{t}+D_{x}^{3}\right) f \cdot g_{j}=0, & \text { for } j=1,2, \cdots N \\ D_{x}^{2} f \cdot f=2 \sum_{1 \leq j<k \leq N} c_{j k} D_{x} g_{j} \cdot g_{k}, & \end{cases}
$$

which is transformed into the ordinary form

$$
\frac{\partial}{\partial t} v_{j}+6\left[\sum_{1 \leq j<k \leq N} c_{j k}\left(D_{x} v_{j} \cdot v_{k}\right)\right] \frac{\partial v_{j}}{\partial x}+\frac{\partial^{3} v_{j}}{\partial x^{3}}=0,
$$

through the transformation

$$
v_{j}=\frac{g_{j}}{f} .
$$

The multisoliton solution to equation (3) is expressed in the same form as that of the coupled modified $\mathrm{KdV}$ equation [5]:

$$
\begin{aligned}
f & =\operatorname{pf}\left(a_{1}, a_{2}, \cdots, a_{L}, b_{1}, b_{2}, \cdots, b_{L}\right), \\
g_{j} & =\operatorname{pf}\left(d_{0}, a_{1}, a_{2}, \cdots, a_{L}, b_{1}, b_{2}, \cdots, b_{L}, c_{j}\right), \text { for } j=1,2, \cdots, N,
\end{aligned}
$$

where the elements of Pfaffians are the same as those of the modified $\mathrm{KdV}$ equation except the element

$$
\operatorname{pf}\left(b_{\mu}, b_{v}\right) \equiv-\frac{c_{j k}}{p_{\mu}^{2}-p_{v}^{2}}, \quad\left\{\begin{array}{l}
b_{\mu} \in B_{j} \\
b_{v} \in B_{k}
\end{array}\right\}, \quad\left(c_{j k}=c_{k j}\right),
$$

is replaced by

$$
\operatorname{pf}\left(b_{\mu}, b_{v}\right) \equiv-\frac{c_{j k}}{p_{\mu}+p_{v}}, \quad\left\{\begin{array}{l}
b_{\mu} \in B_{j} \\
b_{v} \in B_{k}
\end{array}\right\}, \quad\left(c_{j k}=-c_{k j}\right) .
$$

In conclusion we have shown that soliton-solutions of the following equations are expressed by Pfaffians.

(i) Discrete-time Toda equation of BKP type

$$
\begin{aligned}
& W_{n}^{m+1}-W_{n}^{m-1}=\log \frac{\delta^{2}\left[\exp \left(W_{n+1}^{m}-V_{n}^{m}\right)+\exp \left(V_{n}^{m}-W_{n}^{m}\right)\right]+\left(1-2 \delta^{2}\right)}{\delta^{2}\left[\exp \left(W_{n}^{m}-V_{n-1}^{m}\right)+\exp \left(V_{n-1}^{m}-W_{n-1}^{m}\right)\right]+\left(1-2 \delta^{2}\right)}, \\
& V_{n}^{m+1}-V_{n}^{m}=W_{n+1}^{m}-W_{n}^{m} .
\end{aligned}
$$

(ii) Modified Toda equation of BKP type

$$
\begin{aligned}
& \frac{d}{d t} \log \frac{\beta+V_{n}}{\beta-\alpha\left(I_{n}+I_{n-1}\right)}=I_{n}-I_{n+1}, \\
& \frac{d}{d t} I_{n}=V_{n-1}-V_{n} .
\end{aligned}
$$


(iii) Coupled modified $\mathrm{KdV}$ equation

$$
\frac{\partial}{\partial t} v_{j}+6\left[\sum_{1 \leq j<k \leq N} c_{j k} v_{j} v_{k}\right] \frac{\partial v_{j}}{\partial x}+\frac{\partial^{3} v_{j}}{\partial x^{3}}=0, \quad j=1,2, \ldots, N .
$$

(iv) Coupled modified $\mathrm{KdV}$ equations of derivative type

$$
\frac{\partial}{\partial t} v_{j}+6\left[\sum_{1 \leq j<k \leq N} c_{j k}\left(D_{x} v_{j} \cdot v_{k}\right)\right] \frac{\partial v_{j}}{\partial x}+\frac{\partial^{3} v_{j}}{\partial x^{3}}=0, \quad j=1,2, \ldots, N .
$$

\section{REFERENCES}

1. Y. Ohta, R. Hirota, S. Tsujimoto and T. Imai, Casorati and discrete Gram type determinant representations of solutions to the discrete KP hierarchy in bilinear form, $J$. Phys. Soc. Jpn. 62 (1993), 1872-1886.

2. T. Miwa, On Hirota's difference equations, Proc. Japan Acad. Ser. A Math. Sci. 58 (1982), 9-12.

3. Satoshi Tsujimoto and Ryogo Hirota, Pfaffian representation of solutions to the discrete BKP hierarchy in bilinear form, J. Phys. Soc. Jpn. 65 (1996), 2797-2806.

4. Masataka Iwao and Ryogo Hirota, Soliton solution of a modified KdV equation, $J$. Phys. Soc. Japan 66 (1997), 577-588.

5. Masataka Iwao and Ryogo Hirota, Soliton solution of a coupled derivative modified KdV equation, J. Phys. Soc. Japan 69 (2000), 59-72. 